\title{
INTEROPERABLE DOMAIN MODELS: THE ISO LAND ADMINISTRATION DOMAIN MODEL LADM AND ITS EXTERNAL CLASSES
}

\author{
C.H.J. Lemmen ${ }^{\text {a, }}{ }^{*}$, P.J.M. van Oosterom ${ }^{\text {b }}$, H.T. Uitermark ${ }^{c}$, J.A.Zevenbergen ${ }^{\text {a }}$, A.K. Cooper ${ }^{d}$ \\ ${ }^{\text {a }}$ Faculty of Geo-Information Science and Earth Observation (ITC), University of Twente, \\ Hengelosestraat 99, 7514 AE Enschede, The Netherlands - (Lemmen, Zevenbergen)@itc.nl \\ ${ }^{\mathrm{b}}$ Research Institute for the Built Environment, Delft University of Technology, \\ Jaffalaan 9, 2628BX Delft, The Netherlands - P.J.M.vanOosterom@tudelft.nl \\ ${ }^{\mathrm{c}}$ Deventer, The Netherlands - Harry.Uitermark@planet.nl \\ ${ }^{\mathrm{d}}$ Council for Scientific and Industrial Research (CSIR), \\ Meiring Naudé Road; Brummeria; Pretoria; South Africa - ACooper@csir.co.za
}

KEY WORDS: LADM, SDI, Domain Models, Interoperability, Land Administration

\begin{abstract}
:
This paper provides a brief overview of one of the first spatial domain standards: a standard for the domain of Land Administration (LA). This standard is in the draft stage of development now (May 2011). The development of domain standards is a logical follow up after domain-independent standards, which are available now in the area of geo-information processing. The Land Administration Domain Model (LADM) provides a conceptual schema with three basic packages with a limited scope: parties, rights (and restrictions/responsibilities) and spatial units. Certain classes are outside the scope but can be referred to. An important aspect in the development of a coherent (Spatial) Information Infrastructures - (S)II is that the various standardized domain models are reusing the same model patterns as solutions for the same situations.

In this paper the LADM and its external classes are briefly presented. It outlines the advantages of standardized domain models in the development of (S)II and the importance of LA as an authentic register, in relation to other authentic registers, such as for addresses, population, companies, topography, or buildings. This will be illustrated with the Dutch case of authentic registers.
\end{abstract}

\section{INTRODUCTION}

After the development of domain-independent standards for spatial and temporal schemas for spatial features (including metadata standards), a next step is now the standardization of domain-specific standardized models, as a basis for standardized (Spatial) Information Infrastructures - (S)II, also know as the geoweb - development. Examples include International Standards for land cover (ISO 19144-2), Land Administration (LADM, ISO 19152), and Addressing (ISO 19160), all within ISO TC211, or CityGML (from the OGC, which includes topographic features such as buildings, roads, water and earth surface elevation) or GeoSciML (also from OGC) and both based on GML3 (ISO 19136). Other ISO TCs are also working on spatial domain standards; e.g. on Air Quality (TC 146), Water Quality (TC 147), Soil Quality (TC 190), Geotechnics (TC 182), Geosynthetics (TC 221), Fisheries and Aquaculture (TC 234) or some more specific, the well used standards such as the Geographic Data Files (GDF 5.0, ISO 14825 from TC 204) or the Industry Foundation Classes (IFC ISO 16739 for building models from TC 184). At the European level, the INSPIRE directive has identified 34 different geo-information themes, including cadastral parcels, which should be (and are currently being) harmonized.

The Land Administration Domain Model (LADM), ISO DIS 19152, provides an abstract, conceptual schema with basic packages related to (1) parties (people and organizations); (2) rights, responsibilities, and restrictions (ownership and rights, including customary and informal land rights); and (3) spatial units (parcels, customary areas, buildings and networks). The last package includes the surveying and representation subpackage (linked to ISO 19156, Observations and Measurements). LADM enables the combining of Land Administration information from different sources (i.e. organizations) in a coherent manner.

Of course, the scope of the LADM (or any other model) is limited and does not model the whole world. However, certain object classes outside the scope are relevant and should be referred to, but are expected to be defined elsewhere. People and organizations can be referred to as an external class in LADM. This is the case when population registers or company registers are used as an external reference. For land right holders living outside the LA area, an LADM internal class can be used (e.g. people living abroad). The standardization of address data, taxation data, land use data, land cover data, valuation data, physical utility network data, and archive data, is outside the scope of LADM. However, LADM provides external classes for these data sets, which indicate what data set elements LADM expects from these external sources, if available. In LADM for all external classes the stereotype <<blueprint>> is used. This indicates that this object class (and associated classes) are defined somewhere else and should have at least a certain set of attributes. This approach allows the introduction of authentic

\footnotetext{
* Corresponding author.
} 


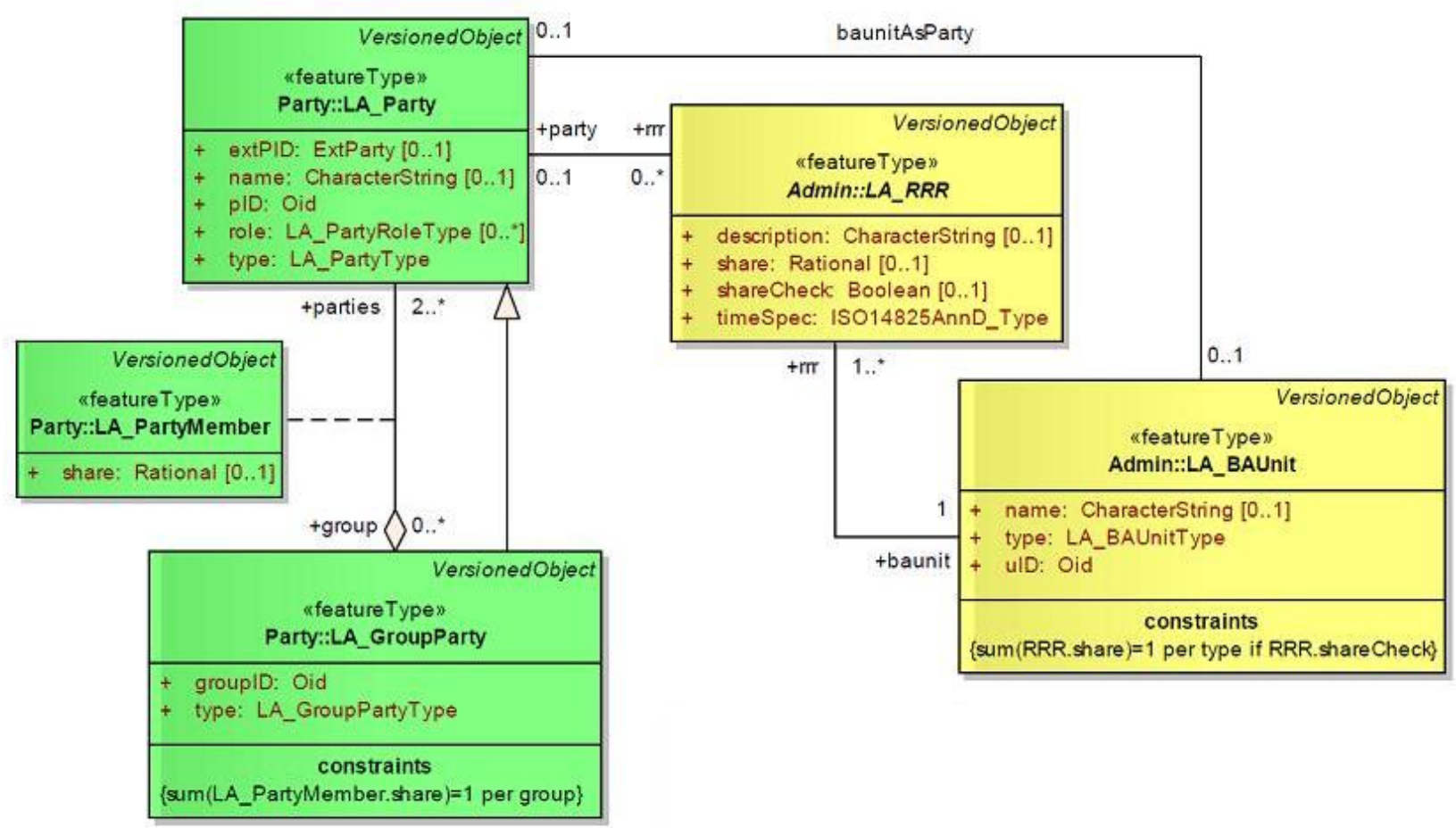

Figure 1, LADM Party package classes, Lemmen et al (2010a)

registers. This paper starts with a brief introduction on the LADM in section 2, with its system boundary in section 3 .. This includes the scope of the draft standard. Then the external classes are presented in section 4. An illustration with the Dutch authentic registers follows in section 5. Finally, the paper summarizes the main findings and provides indications of future work in section 6 .

\section{THE LAND ADMINISTRATION DOMAIN MODEL}

The standardized Land Administration Domain Model (LADM), covers land registration and cadastre in a broad sense (spatial and administrative components, source documentation included)..Such a Land Administration Domain Model (ISO as well as land, and elements above and below the surface of the earth). 3D Cadastres are covered in such a way that they seamlessly integrate with exiting $2 \mathrm{D}$ registrations.

It includes agreements on data about administrative and spatial units, land rights in a broad sense and source documents (e.g. titles, deeds or survey documentation). The rights may include real and personal rights as well as customary and informal rights; the latter can be included as 'social tenure relationships' in the Social Tenure Domain Model (STDM). Restrictions and responsibilities can be similarly represented to document the relationships between people and land.

LADM describes the data contents of Land Administration in general, based on a practical approach. The roots are, amongst others, in FIG's Cadastre 2014 (Kaufmann and Steudler, 1998), in Fourie (1998), and in Henssen (1995). Implementation of LADM can be performed in a flexible way; the standard can be extended and adapted to local situations. Existing standards have been re-used in LADM, particularly the ISO 19100 series from ISO/TC 211, which has an extensive process for increasing harmonization between its standards, (2011a); Van Oosterom et al (2006); Lemmen et al (2010a, 2010b)) underpins existing conventional Land Administration systems. These conventional systems take into account conventional legal forms of evidence and are in principle parcel based. This means that they only cover a portion of all forms of land tenure. Globally there are many examples where the land use rights of informal settlement residents, slum dwellers, families and groups living under customary tenure, indigenous people, pastoralists, or refugees are not capable of being integrated into a conventional Land Administration system.

The draft standard for the Land Administration Domain defines a reference model covering basic information-related components of Land Administration (including those over water

such as through an integrated UML model for the technical content of all its standards, integrated terminology and a proposed programme for configuration management of all its standard (Coetzee et al 2011). LADM also includes references to the standard, ISO 19156, Geographic information Observations and Measurements, which is under development within ISO/TC 211.

The three main packages of the LADM, the Party package, the Administrative package, and the Spatial Unit package are as follows. The main class of the Party package is the class LA_Party, and its specialization LA_GroupParty. There is an optional association class LA_PartyMember (Figure 1). Parties are persons, or groups of persons, or juridical persons, that compose an identifiable single (legal) entity. A juridical person may be a company, a municipality, the state, or a church community.

The Administrative package concerns the abstract class LA_RRR (with its three concrete subclasses LA_Right, LA_Restriction, and LA_Responsibility), and class LA_BAUnit (an abbreviation for 'basic administrative unit'), 


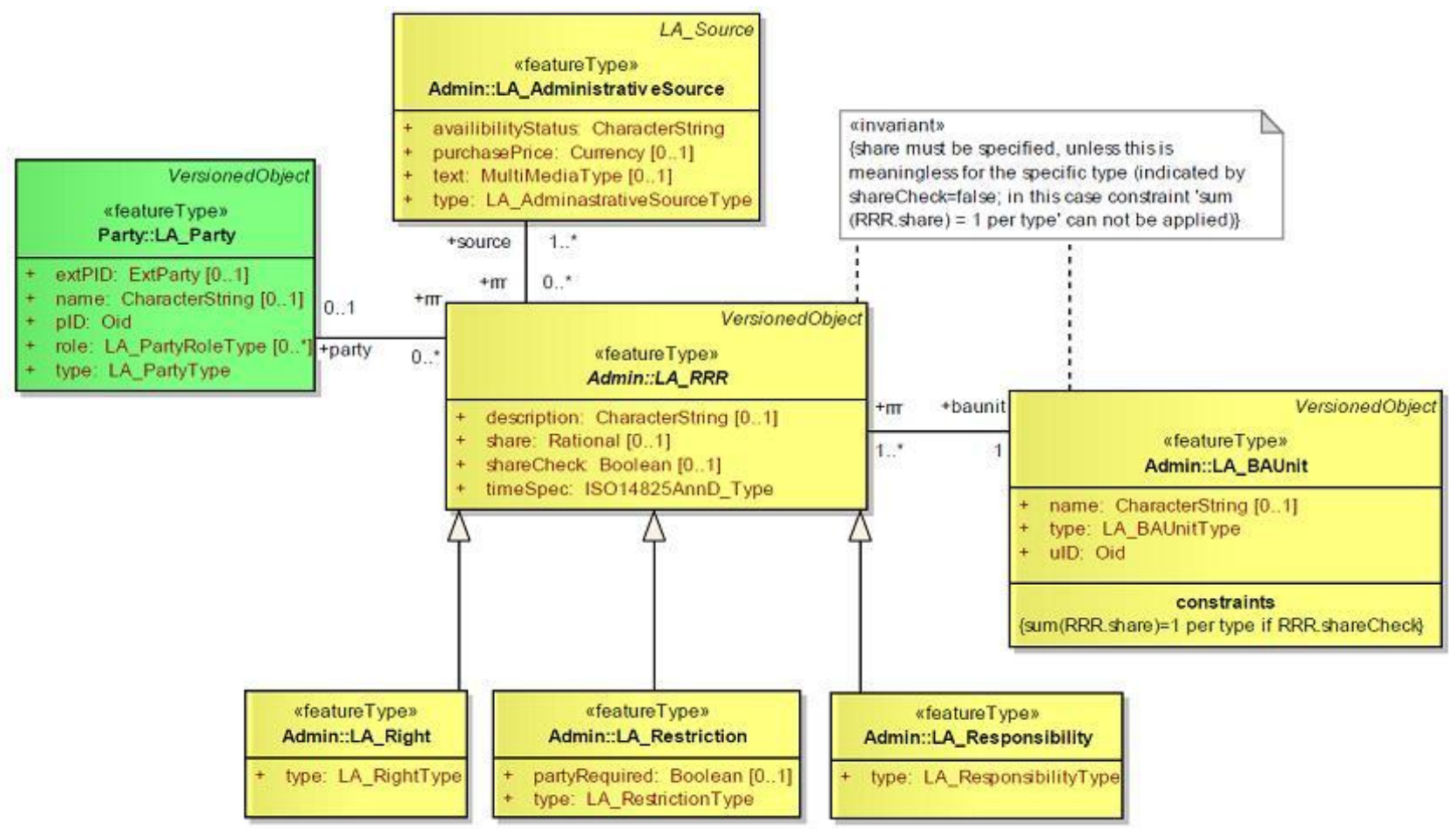

Figure 2, LADM Administrative package classes, Lemmen et al (2010a)

see Figure 2. A 'right' is a formal or informal entitlement to own, to do something, or to refrain from doing something. Examples are: ownership right, customary right, or informal right. Rights may be overlapping. A 'restriction' is an obligation to refrain from doing something; e.g. it is not allowed to build within 200 meters of a fuel station. A 'responsibility' is a formal or informal

obligation to do something; e.g. the responsibility to clean a water canal or to maintain a road. A 'baunit' is an administrative entity consisting of zero or more spatial units against which the same (set of one or more) rights, responsibilities or restrictions are associated, as included in a LA system. A 'basic administrative unit' may be a 'party' because it may hold a right of easement over another spatial unit, see Figure 1.

The Spatial Unit package concerns the classes LA_SpatialUnit, LA_SpatialUnitGroup, LA_Level, LA_LegalSpaceNetwork, LA_LegalSpaceBuildingUnit, LA_RequiredRelationshipSpatialUnit (Figure 3) and the classes in the Surveying and Representation Subpackage. A 'spatial unit' is based on a point (or, multi-point), a line (or, multi-line), representing a single area (or, multiple areas) of land (or water) or, more specifically, a single volume of space (or, multiple volumes of space).

Spatial units are a flexible concept of representing reality and may be described in text, or based on a single interior point, or as a collection of lines or polygons, or as a $3 \mathrm{D}$ volume with or without topology. A 'spatial unit group' is a group of spatial units; e.g.: spatial units within an administrative zone, or within a planning area (Lemmen et al, 2010b).

A 'level' is a collection of spatial units with a geometric or thematic coherence; for example: 1 . Urban and rural level, or 2. Forest, railway or road level, or 3. Formal, informal, or 4. A level with point based spatial units, a second level with line based spatial units, and a third level with polygon based spatial units. The different spatial units may be arranged to 'information levels' according to the laws by which they are defined.

\section{THE SYSTEM BOUNDARY OF THE LADM}

The LADM is organized into several packages. It is likely that more packages will be developed. Besides being able to present/document the model in comprehensive parts, another advantage of using packages is that it is possible to develop and maintain these packages in a more or less independent way. Domain experts from different countries could further develop each package based on LADM.

LA modeling depends a lot on the scope of the models; e.g. if one Land Administration model includes a person registration and the other model just refers to a person, then the two models may look different, but the intentions are the same. Only the system boundary of the involved models is different.

However, the boundary of the LADM is arbitrary in a certain sense. Perhaps, also (some of the) current packages of the model should be considered as separate models outside the LADM; or more models inside the LADM may be needed. The LADM packages have been introduced in section 2 . The following is a list of classes, or packages of classes that are related to, but outside LADM:

Spatial (coordinate) reference system. It should be noted that the physical implementation of a reference system is part of

conventional cadastral systems. There can be more then one reference system for different parts of the territories where such systems are implemented; e.g. one local coordinate system per village. Spatial reference systems are the basis for getting nationwide cadastral spatial data available. In LADM the Spatial Reference System (SRS) appear via the GM_Point attribute in the LA_Point, LA_Spatial Unit and 


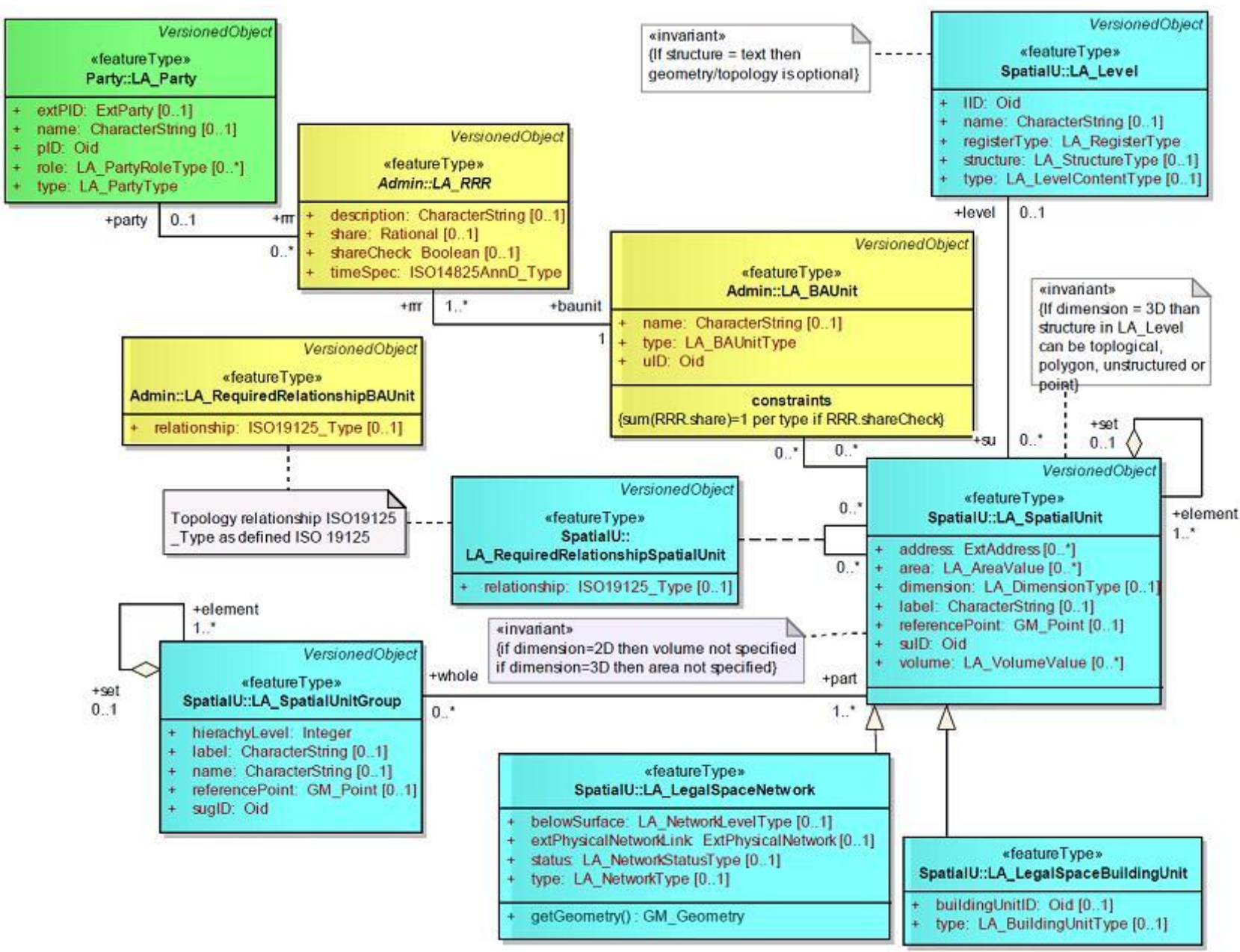

Figure 3, LADM spatial unit package classes, Lemmen et al (2010a)

LA_SpatialUnitGroup; via the GM_Curve attribute in LA_BoundayFace and via the GM_Surface attribute in LA_BoundayFace classes. In fact those attributes are re-used from ISO 19111, spatial referencing by co-ordinates; and ISO 19107 spatial schema - GM_point and GM_MulitCurve and GM_Surface are defined here. For this reason spatial reference systems are excluded from LADM, as well feature types for spatial data.

Ortho photos, satellite imagery, and Lidar and elevation models. Here it should be noted that ortho photo's and satellite imagery may be very well used as basis for data acquisition in the field of cadastral boundary data (Lemmen and Zevenbergen, 2010). The cadastral boundaries can be identified in the field on top of such images. The imagery source can be described in attributes in the LA_SpatialSource class, and in the DQ_Element attribute which is part of the LA_VersionedObject ${ }^{*}$ class. The images itself may inlcuded in LADM using LA_SpatialSouce.

* LA_VersionedObject is a superclass. Classes LA_Party, LA_GroupParty, LA_PartyMember, LA_Mortgage, LA_RRR, LA_BAUnit, LA_SpatialUnit, LA_SpatialUnitGroup,

LA_RequiredRelationshipSpatialUnit,

LA_RequiredRelationshipBAUnit, LA_Level, LA_BoundaryFaceString, LA_BoundaryFace, and LA_Point are
Topography (planimetry). Again this is considered to be a domain in it itself. Topographic maps (or databases with topographic data) may be used as a basis for cadastral boundary data acquisition and maintenance; the topographic maps/data can be used as spatial source (as evidence from the field).

- Geology, geo-technical and soil information. This is relevant information in relation to mining and land use (agricultural) management - those are domains in itself. The LADM supports the inclusion of attributes as results of data collection processes on geology, soil, etc. In this way a Land Administration for mining may be built up; this would include concessions and exploration companies as parties.

- (Dangerous) pipelines and cable registration. This concerns the physical registration of cables and pipelines good external references are possible here using the extPhysicalUtilityNetworkID attribute under LA_LegalSpaceUtilityNetwork as subclass from LA_SpatialUnit. LADM is about legal space in 3D. This includes of course the registration of access to utilities as restrictions to other land rights of other parties (rights of way,

all subclasses from LA_VersionedObject. LA_VersionedObject has attributes for the management of versions of objects in LADM and for the management of data quality. 
encumberances, servitudes). It is very important to recognize that legal space around a utility cable, pipeline does not necessarily coincide with the physical space of a cable or pipeline in a network. Utilities can be invisible - antennas should "see" each other for signal transmissions. For all utilities a 3D partition of space is very helpful in representation. This may also include access to e.g. airports.

Address registration (including postal codes). Standards for addresses are under development as ISO 19160. Addresses in LADM concern spatial unit addresses ("object" addresses) but of course parties can have addresses ("subject" addresses) - but in LADM those addresses are considered to be available via extParty class: this is the population register, or the company register. Of course the external address class as introduced here below can be included in a LADM implementation.

Building registration, Both (3D) geometry and attributes (permits), this concerns the physical registration. The registration of legal space in 3D is included in LADM. Legal space does not necessarily coincide with the physical space of a building.

Natural person registration - the authentic person data are considered to be in the population register: name, date of birth, person address, sex, etc.

Non-natural person (company, institution) registration. Same for typical attributes of non natural persons, e.g. companies.

Polluted area registration. This may be subject of registration. In fact the responsibilities as a consequence from such pollution or the restrictions following from it can be included in LADM. This domain of polluted area registration could be an extension in the future. Something similar may be valid for energy labels for buildings.

Mining right registration. See before under item 4.

Cultural history, (religious) monuments registration. This can be included using local attributes defined for this purpose.

Fishing/hunting/grazing right registration.

Ship- and airplane (and car) registration. Even car registration comes in here in case of distinction between movables and immovables. But ships and airplanes may be defined as immovable - because there can be a mortgage established.

Most of the registration related issues identified here can be included in LADM using its basic structure and options of local extensions. In all cases the processes will not be the subject of modeling, only the outcomes of processes (steps) can be included in such extended local versions of LADM.

\section{LADM EXTERNAL CLASSES}

The (external) databases with party data, address data, valuation data, land use data, land cover data, physical utility network data, archive data, and taxation data is outside the scope of the LADM. However, LADM provides stereotype classes for these data sets, which indicate what data set elements LADM expects from these external sources, if available. Figure 4 presents an overview on external classes in relation to the LADM core classes. The classes are briefly introduced here. Classes which are outside the scope of the LADM (e.g. ExtPersons, ExtAddress, ExtTaxation, Extvaluation, ExtUsage) are represented as <<blueprint >> stereotype classes. They do not have the 'LA_' prefix, but they do give an exact definition of what the LADM is expecting of these external classes.

The Class ExtParty is a <<blueprint >> class for an external registration of parties. This can be a link to a population register or to a chamber of commerce with a company register. The attributes of ExtParty are:

extAddressID: this is the identifier, pointing to the external address;

fingerprint: the fingerprint of the external party;

name: the name of the external party;

partyID: this is the identifier of the external party;

photo: the photo of the external party; and:

signature: the signature of the external party.

It should be noted here that LADM itself also includes its own classes for the representation of (group) Parties. This is needed for people living abroad: people not living in the area where the Land Administration applies. It may also be needed in case a population register or company register does not yet exist.

Class ExtAddress is a <<blueprint $>>$ class for an external registration of addresses (an address being a direction for finding some location). The attributes of ExtAddress as proposed in Annex $\mathrm{K}$ of the draft international standard are:

addressAreaName: the address area name of the external address;

address;

addressCoordinate: the coordinates of the external

- addressID: the identifier of the external address;

buildingName: the building name of the external address;

buildingNumber: the building number of the external address;

- $\quad$ city: the city of the external address;

country: the country of the external address;

postalCode: the postal code of the external address;

postBox: the post box of the external address;

state: the state of the external address;

streetName: the street name of the external address.

The INSPIRE address specifications (INSPIRE 2010) may also be used, or an ISO standard on addresses. The ISO 19160 project team has documented well the review of existing addressing standards and provided the recommendations that describe the addressing standardization requirements for ISO 19160 (ISO 2011b). These include addressing terminology, conceptual models, address assignment schemes, quality management and rendering addresses on postal items, maps, graphic displays, etc. Address co-ordinates can be used for geo coding services in combination with reference points and labels in spatial units and spatial unit groups and points. In LADM there is a reference from spatial unit to external address: the object address and from Party to external address: the subject address. This is because the right holder does not need to reside on the spatial unit - building, or apartment.

Class ExtLandUse is a <<blueprint >> class for the external registration of land use data; land use is an arrangement, activity or input people undertake in certain land cover type, to produce, change or maintain it. ExtLandUse is associated to class LA_SpatialUnit. Like taxation it is normal that land use is integrated in Land Administration and cadastre. A similar discussion took place for land use as for taxation. With a similar result. The attribute of ExtLandUse is:

type: the type of land use.

The LADM is designed in such a way that the inclusion of land use can be integrated later.

Class ExtLandCover is a <<blueprint $>>$ class for the external registration of land cover data; land cover is the observed 


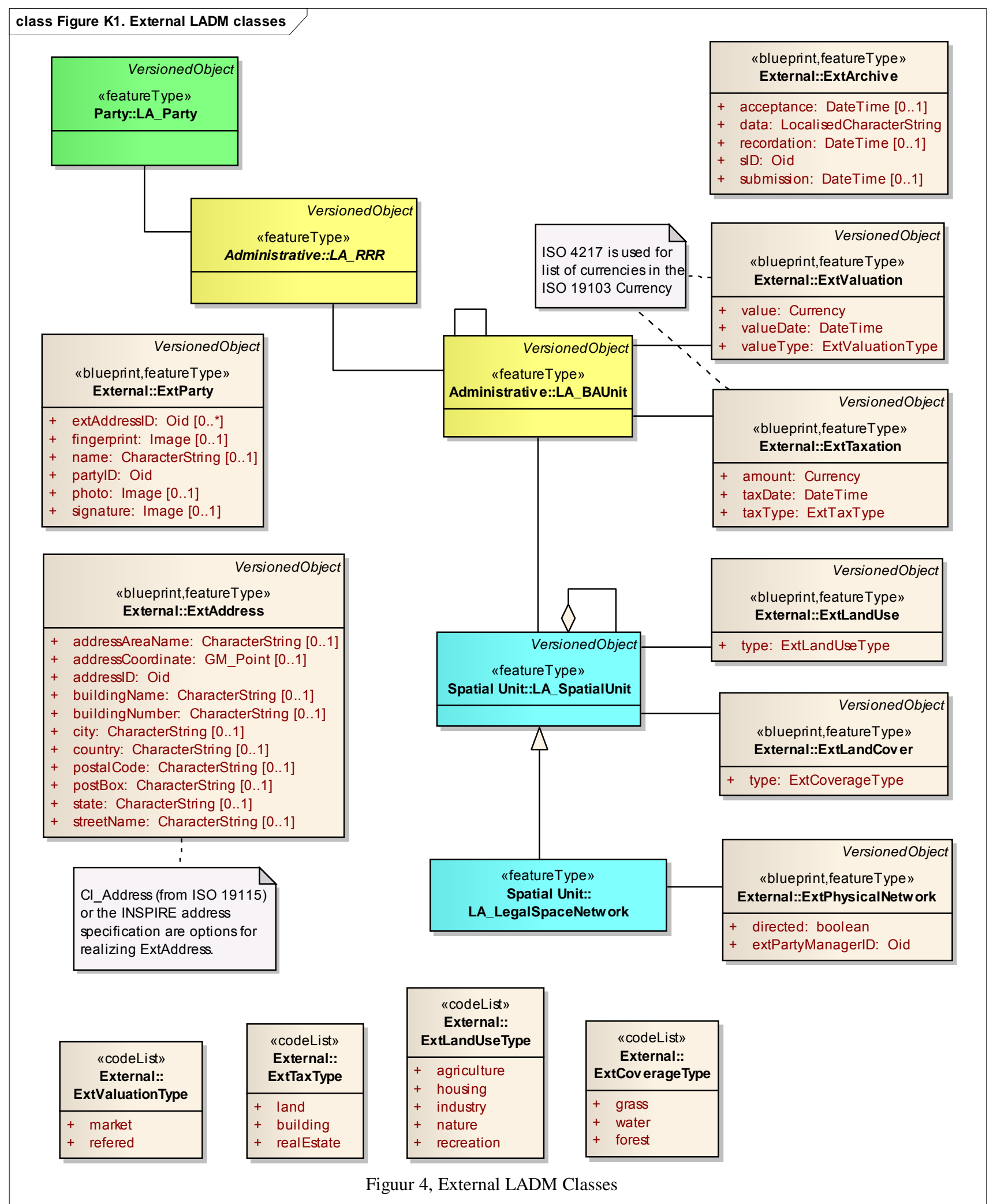

(bio)physical cover on the earth's surface. ExtLandCover is associated to class LA_SpatialUnit. The attribute of ExtLandCover is:

type: the type of land cover.

See the discussion on land use above - this is valid again for land cover, but here also the ISO 19144 Land Cover standard applies.
Class ExtValuation is a $<<$ blueprint $>>$ class for the external registration of valuation data. ExtValuation is associated to class LA_BAUnit. The attributes of ExtValuation are:

- value: the value of the valuation;

- valueDate: the date of the valuation;

- valueType: the valuation type.

Again this is seen as an external process. The data resulting from this process can be linked to LADM. 
Class ExtTaxation is a <<blueprint $>>$ class for the external registration of taxation data. ExtTaxation is associated to class LA_BAUnit. The attributes of ExtTaxation are: amount: the amount of taxation; taxDate: the date of taxation; taxType: the tax type.

It is common practice is some countries that taxation can be included in Land Administration and cadastre. The inclusion of taxation has been considered in the development of LADM. The conclusion was that the valuation and required spatial and non spatial data and also the determination of tax values is a domain in it self and therefore outside the scope of the LADM. However, the LADM is designed in such a way that the (S)II based integration with taxation can be realized.

Class ExtPhysicalUtilityNetwork is a <<blueprint $>$ class for the external registration of mapping data of utility networks. ExtPhysicalUtilityNetwork is associated to class LA_LegalSpaceUtilityNetwork. The attributes of ExtPhysicalUtilityNetwork are:

- directed: the flow direction, fixed or not; managerID: the organization responsible for the utility network.

Class ExtArchive is a <<blueprint >> class for the external registration of sources. The attributes of ExtArchive are: authority; acceptance: the date of force of law of the source by the - data: the content of the source;

recordation: the date of registration (recordation) of the source by registering authority;

sID: the identifier of the source;

- submission: the date of submission of the source by a party.

This is the link to archives. The ISO standard for archives which is reused in LADM. Attributes submission, acceptance, recordation allow for links to workflows. This means the status of a transaction can be followed. This is relevant to know to avoid double transactions.

\section{LADM WITHIN THE INFORMATION INFRASTRUCTURE}

Information and Communication Technology (ICT) offers many opportunities for improving the performance of government and business. Areas which may profit include education, safety, health care, international co-operation, economic efficiency (integrated value chains, business-process management, and reduction in administrative overhead), prevention and detection of fraud, or accident and disaster management. ICT trends such as ubiquitous access, smart objects, open source, increased bandwidth, interoperability, and data-exchange standards will result in new business models. New perspectives are opened up by options like increased location independence, high-quality online services based on immediate access to all required data, use of identified objects available for process control, integration within business chains and government organizations, and increased e-shopping.

Groothedde et al (2008) argued that the information content within the (Spatial) Information Infrastructure (SII) consists of several key registers or databases and that it is therefore important to define what contents belong to what register. The different databases within the (S)II are related, i.e. there are references in the content from one database to another. As the databases are maintained by 'independent' organizations care has to be taken when information is updated that related databases are informed (in order to trigger potential related updates elsewhere). The fundamental question is: 'How to maintain consistency between two related distributed systems in case of updates?'. Assume that System A refers to object X in System B (via object id B.X_id), now the data in System B is updated and object 'X_id' is removed. As long as System A is not updated the reference to object $X$ should probably be interpreted as the last version of this object available. Note that the temporal aspect has an important role in and between the systems! The true solution is also updating system A and removing the reference to object $X$ (at least at the 'current' time). How this should be made operational will mainly depend on the actual situation and systems involved. It might help to send 'warning/update messages' between systems, based on a subscription model of the distributed users/systems. An extremely important aspect of the future (S)II, in which (related) objects can be obtained from another database/organization (instead of copied), is that of 'information assurance'. Though the related objects, e.g. persons in case of a cadastral system, are not the primary purpose of the registration, the whole cadastral 'production process' (both update and delivery of cadastral information) does depend on the availability and quality of the data at the remote server. Some kind of 'information assurance' is needed to make sure that the primary process of the cadastral organization is not harmed by disturbances elsewhere. In addition, remote (or distribute) systems/users might not only be interested in the current state of the objects, but they may need an historic version of these objects; e.g. for taxation or valuation purposes. So even if the organization responsible for the maintenance of the objects is not interested in history, the distributed use may require this (as a kind of 'temporal availability assurance'). It is clear that this can have a serious impact on the data management at the side of registering organization.

Other topics have a strong relationship in the sense that these (physical) objects may result in legal objects ('counterparts') in the Land Administration. For example, the presence of utility cables or pipelines can also result in a restriction area or space (2D or 3D) in the Land Administration. However, it is not the cable or pipeline itself that is represented in the cadastral system; it is the legal aspect of this. The fact that these 'physical' objects are so closely related to the 'legal' objects within the Cadastre, also implies that it is likely that some form of interoperability is needed. When the cables or pipelines are updated, then both the physical and legal representations should be updated consistently (within a given amount of time). This requires some semantic agreement between the 'shared' concepts, or at least the interfaces and object identifiers. In other words these different but related domain models need to be harmonized. As it is already difficult within one domain (such as the cadastral world) to agree on the concepts used and their semantics, it will be even more difficult when we are dealing with other domains. However, we can not avoid this if a meaningful interoperable geoinformation infrastructure has to be developed and implemented.

The basic idea behind information infrastructures is that they provide the tools that give easy access to distributed databases to people who need those data for their own decision making processes. An important advantage of this approach is that it is avoided to make (many) copies and also that there are no 
copies to be maintained, just the source. Although information infrastructures have a substantial component of information technology, the most fundamental asset is the data itself, because without data there is nothing to have access to, to be shared or to be integrated. In the last decade it was understood that the development of information infrastructures not only provided easy access to distributed databases, but also gave good opportunities for re-thinking the role of information supply for the performance of governments. Based on this starting point, the Program 'Streamlining Key Data' of the Dutch government took the lead in the development and implementation of a strategy for restructuring government information in such a way that an electronic government will evolve that:

will only bother the public and the business community with requests for data when this is absolutely necessary

- $\quad$ offers the public and the business community a rapid and good service

can not be misled

- instills the public and the industrial community with confidence

is provided at a cost that is not higher than strictly necessary.

Jointly with five other government registers, the property register, cadastral map and topographic map of the Dutch Cadastre, Land Registry and Mapping Agency ('Kadaster') have been formally appointed in 2002 as 'key registers' of the governmental information infrastructure. The key registers will be the core of a system of so-called authentic registers, which might be any register that is maintained by a single government body and used by many others as the authentic source of certain data. If a register is formally designated as an authentic register, all other government organizations are strictly forbidden to collect the same data by themselves. In their budget allocation they will not find any money for data collection at this point. The Program 'Streamlining Key Data' concentrates on two goals: 1 . the communal use of data: in principle data would be collected on one occasion, and repeatedly used for the implementation of series of laws, and 2. the joint use of data: data from different registrations (organizations) required for the performance of a specific government duty would be combined in one application.

An authentic register is defined in the Program as 'a high quality database accompanied by explicit guarantees ensuring for its quality assurance that, in view of the entirety of statutory duties, contains essential or frequently-used data pertaining to persons, institutions, issues, activities or occurrences and which is designated by law as the sole officially recognized register of the relevant data to be used by all government agencies and, if possible, by private organizations throughout the entire country, unless important reasons such as the protection of privacy explicitly preclude the use of the register'. The Legislation has been created for the designation of the following registers:

- Municipal Personal Records Database - Population
Register
- $\quad$ Cadastre (Parcels and Rights)
- $\quad$ Company Key Register ('New Trade Register')
- $\quad$ Addresses, Buildings, and
- $\quad$ Topography.

The Dutch Cadastre's current strategic objectives might be reformulated. It aims for the best possible performance of current public duties and the promotion of innovation and knowledge by adopting a leading role in their evolution in response to societal developments. Strategic sub-objectives are: investigation of evolution towards more legal evidence of registered data (a positive land-registration system with state guarantee)

- $\quad$ introduction of a 3D land register

ambition to adopt role as centre for a range of key registers

provision of more complete in-sight into private and public legal status of registered property

achieving a substantial role in organizing information needs of the property market chain

provision of appropriately linked set of object-oriented topographic and geographic datasets, mutually consistent with respect to change and $\mathrm{z}$ )

fulfillment of pivotal role in geometric infrastructure ( $\mathrm{x}, \mathrm{y}$

acceptance of prominent EU partner role in harmonizing registered-property law, land registration, and cadastres

development of flexible land-planning instruments suitable for use in realizing a variety of societal spatial objectives.

\section{CONCLUSIONS}

The Land Administration Domain is at stage Draft International Standard. It's system boundary is well defined, but arguably somewhat arbitrarily. But a scope is needed, also for other domains. This allows for implementation of clear responsibilities in the context of Spatial Information Infrastructures (SII). Other standards, from ISO or else, have or are being defined for the neighboring domain models, enabling meaningful shared use in applications or even integration, that is, 'one registry partly builds on the content of another registry'.

It will be made easier to establish links between data obtained online and to import data into user' automation systems from data providers. Data providers may recognise that their activities form a kind of 'supply chain' with activities performed in co-operation with other parties to meet society's expectations. A very good example of using and integrating spatial information in the SII is the combination of the (agricultural) Integrated Administration and Control System (IACS), including a Land Parcel Identification System (LPIS) with its own LPIS Core Model (LCM) with the LADM (Inan et al., 2010, ISO 2011a, Annex G). The SII can be used to share the relevant pieces of information between LPIS and LADM systems. However, without standardization, the involved models and systems in different regions and countries will definitely hamper implementations of SII at both national and international (EU) level. Therefore these standardization efforts are crucial to make the SII really work.

It is important to realize that we are at the very early stage of creating and using the SII. Our initial standardization efforts and systems implementations will need to be evaluated based on the actual experience when using (integrated) information form the SII. Neighbour domains might need fine-tuning and as a result of this our standards and implementations will for sure need future revisions. In the realization and use of SII, standards and systems are enables, but many other factors also play an important role: legal, organizational and technical factors to name a few. It is also important that these are well addressed. 


\section{References from Journals:}

Inan, H.I., V. Sagris, W. Devos, P. Milenov, P. van Oosterom, and J. Zevenbergen (2010). Data model for the collaboration between land administration systems and agricultural land parcel identification systems. In: Journal of Environmental Management, Volume 91, 12, pp. 2440-2454.

Van Oosterom, P. J. M., C. H. J. Lemmen, T. Ingvarsson, P. van der Molen, H. Ploeger, C. W. Quak, J. E. Stoter and J. A. Zevenbergen (2006). "The core cadastral domain model." Computers, Environment and Urban Systems 30(5): 627-660.

\section{References from Books:}

Groothedde, A., C. H. J. Lemmen, P. van der Molen and P. J. M. van Oosterom (2008). A standardized land administration domain model as part of the (spatial) information infrastructure. Creating spatial information infrastructures. Towards the spatial semantic web. P. J. M. van Oosterom and S. Zlatanova. Boca Raton, Florida, CRC Press. Taylor \& Francis Group: 129-150.

Lemmen, C. H. J. and J. A. Zevenbergen (2010). First experiences with a high-resolution imagery-based adjudication approach in Ethiopia. Innovations in land rights, recognition, administration, and governance. K. Deininger, C. Augustinus, S. Enemark and P. Munro-Faure. Washington D.C., The World Bank: 96-104.

\section{References from Other Literature:}

Coetzee S., S. Cox and J. Herring (2011) Configuration management of a system of interdependent standards. The 7th International Conference on Standardization and Innovation in Information Technology (SIIT2011), Berlin, Germany, 28 - 30 September 2011 (submitted).

Fourie, Clarissa, (1998), An integrated geo-information system with emphasis on cadastre and land information systems for decision makers in Africa, working document for the expert group meeting held at United Nations Economic Commission for Africa, Addis Ababa, Ethiopia, 23 - 26 November 1998.

Henssen, J. L. G. (1995). Basic principles of the main cadastral systems in the world. Seminar Modern Cadastres and Cadastral Innovations. D. Steudler. Delft, The Netherlands, FIG: 5-12.

INSPIRE (2010). D2.8.I.5 INSPIRE Data Specification on Addresses - Guidelines (version 3.0.1), 177p.

ISO (2011a). ISO/DIS 19152. Draft International Standard (DIS). Geographic information - Land administration domain model (LADM). Geneva, Switzerland, ISO: 110p.

ISO (2011b) TC211 Geographic information/Geomatics, N 3075, Draft Review summary of project 19160, Addressing. ISO/TC 211 Secretariat, Standards Norway: 121p.

ISO (2011c) ISO/DIS 19156. Draft International Standard (DIS). Geographic information - Observations and measurements. Geneva, Switzerland, ISO: 58p.
Kaufmann, J. and D. Steudler (1998). Cadastre 2014. A vision for a future cadastral system. FIG XXI International Congress, Brighton, U.K.

Lemmen, C. H. J., P. J. M. van Oosterom, R. J. Thompson, J. Hespanha and H. T. Uitermark (2010a). The modelling of spatial units (parcels) in the Land Administration Domain Model (LADM). XXIV FIG International Congress 2010. Sydney, Australia.

Lemmen, C. H. J., P. J. M. van Oosterom, C. Eisenhut and H. T. Uitermark (2010b). The modelling of rights, restrictions and responsibilities (RRR) in the Land Administration Domain Model (LADM). XXIV FIG International Congress 2010. Sydney, Australia. 
International Archives of the Photogrammetry, Remote Sensing and Spatial Information Sciences, Volume XXXVIII-4/C21 